\title{
Common label pretraining: Interference and/or facilitation?
}

\section{E. MARSHALL, Carleton University, Ottawa 1, Canada}

Twenty Ss were assigned to each of four verbal pretraining conditions whose proactive effects were assessed in a subsequent discriminative motor task. Ss in Group $R D L$ learned a different verbal response to each of six 24-point random shape stimuli, while Group $R C L$ Ss learned two verbal responses to clusters of three of the stimuli. One control group, ICL, learned two responses to two clusters of three different stimuli, and a final group, $N L$, received no pretraining. Reliable differences obtained between the four treatment groups whose transfer performance rank in order of increasing errors was $R D L, R C L, I C L$, and $N L$.

Several tests of the hypotheses of acquired equivalence and distinctiveness of cues (Dollard \& Miller, 1950) have employed common-label (CL) and/or different-label (DL) treatments during the pretraining or predifferentiation (PD) phase of the investigations (Robinson, 1952; Ellis, Bessemer, Divine, \& Trafton, 1962; Katz, 1963; Ellis \& Muller, 1964; del Castillo \& Ellis, 1968). Equivalence or CL training has required that Ss learn common labels to clusters of two or more stimuli within a test list. Distinctiveness or DL training has required that Ss learn a different label to each stimulus. The majority of these studies have compared the performances of CL and DL groups on recognition or simple discrimination tasks with the performance of a control which has merely observed $(O)$ the critical stimuli during PD training. Under limited conditions of stimulus complexity and response relevance, DL training has facilitated test task performance, while $\mathrm{CL}$ training has resulted in performance inferior to that of the $O$ control. These data have been interpreted as consonant with the supposition that $\mathrm{CL}$ training increases the overall generalization tendencies among the stimuli of the test list, while DL training enhances their discriminability through the acquisition of identical and distinctive verbal response-produced cues, respectively.

Recently, del Castillo \& Ellis (1968) tested the acquired equivalence (AE) and acquired distinctiveness (AD) hypotheses in the setting of a transfer task which required Ss to make new discriminative motor responses to the stimuli of the verbal PD task. Using four visual stimuli which differed along the single dimension of light intensity, the authors found no evidence for $\mathrm{AE}$, although the $\mathrm{AD}$ hypothesis was supported by the superior performance of DL-treatment groups compared with $\mathrm{O}$ controls. One factor which may have contributed to the failure of evidence for $\mathrm{AE}$ (and to the strong evidence for AD within the same design) is the limited appropriateness of the $\mathrm{O}$ control in the $\mathrm{AB}-\mathrm{AC}$ stimulus differentiation paradigm. While the $O$ treatment does familiarize Ss with the stimuli of the transfer task, and may as well allow Ss to form systematic implicit associations to these stimuli (Ellis \& Homan, 1968), it does not give Ss the opportunity to acquire the performance set necessary to the efficient mastery of a subsequent associative task in which the overt paced production of discriminative motor responses is required. Thus, in proceeding from PD to transfer tasks, O-treatment Ss may suffer a performance decrement attributable to differences between task demands. This would at once vitiate the expected superiority of the performance of $O$ Ss to those given the CL treatment, and maximize the superiority of $\mathrm{DL}$ to $\mathrm{O}$ treatments.

The purpose of the present experiment was to explore within the $\mathrm{AB}-\mathrm{AC}$ paradigm the proactive consequences of $\mathrm{DL}$ and $\mathrm{CL}$ training with respect to a control group given irrelevant training (ICL) and an additional control given no training at all (NL). While the ICL control procedure resembles the $O$ treatment in not permitting the separation of the effects of verbal response attributes from other aspects of the PD task, it does improve on the $\mathrm{O}$ treatment in providing control Ss with the opportunity to develop a performance set appropriate to the transfer task. A secondary purpose of the experiment was to discover whether or not the speed of acquisition during $P D$ training is reliably associated with transfer task performance.

\section{METHOD}

Subjects were 80 students enrolled in Introductory Psychology at Carleton University. They were assigned to one of four PD treatment conditions: Ss in Group RDL learned six meaningful CVCs (average $m^{1}=4.41$, according to Noble, 1961 ) to the six stimuli (24-point random shapes generated and scaled for discriminability by Somnapan, 1968) of the subsequent transfer task; Ss in Group RCL learned one of the CVCs to one cluster of three of the same shapes and another CVC from the list to the remaining three shapes. An irrelevant stimulus control group (ICL) learned the same two responses to two clusters of three different random shapes equated for complexity. Within Groups RCL and ICL, S-R pairings were arranged such that each stimulus appeared in a cluster with each. other stimulus an equal number of times. A further control group (NL) received no first-task training. Verbal acquisition for Groups RDL, RCL, and ICL proceeded by the paced paired-associates anticipation method. All Ss were brought to a criterion of three successive blocks of six correct anticipations.

The 20 trials of the paced paired-associates motor task were given by the anticipation-correction method using an apparatus described in detail by Marshall (1968). Ss were informed that on this task a different motor response was to be learned to each of six shapes, and they were told to attempt an anticipation upon each stimulus presentation.

\section{RESULTS}

The mean number of errors per $S$ per trial for Groups RDL, RCL, ICL, and NL over 20 transfer-task trials were $0.84,1.39$ 2.08 , and 2.77 , respectively. The acquisition functions for the four groups maintained the rank order of the overall means on all test trials. An analysis of variance applied to these data yielded significant main effects for Groups $(p<.01$ $F=27.41 ; \quad d f=3,76), \quad$ for Trials $(p<.01 ; \quad F=84.58$ $\mathrm{df}=19,1444$ ), and for the Groups by Trials interaction $(p<.01 ; F=2.45 ; \mathrm{df}=57,1444)$.

Using the critical difference for $t_{05}$ based on the mean square within cells for the entire data matrix, the effects attributable to the number of verbal labels learned to stimuli during PD (RCL-RDL) proved significant only on early transfer trials (Trials 3-10), while effects attributable to the relevancy of stimuli (ICL-RCL) were significant over the middle range of the task (Trials 6-14). Differences attributable to warm-up, learning-to-learn, and other performance-set factors (NL-ICL) occurred significantly first on Trial 5 and last on Trial 19 of the transfer task.

After Ss in Groups RCL, RDL, and ICL were ranked in terms of number of trials to criterion on the PD task, the transfer data for the six slowest and the six fastest learners of each group were subjected to statistical test. The main Groups effect was significant, as in the analysis of the data for all Ss, 
but PD acquisition speed was not a significant variable, nor did it interact reliably with either Groups or Trials, although the overall mean number of errors for fast PD learners (1.33 per $S$ per trial) was slightly lower than the corresponding measure for slow PD learners (1.58 per $S$ per trial).

\section{DISCUSSION}

Four features of the error measures of the transfer task are of interest. First, the superior performance of Group ICL to that of Group NL confirms previous findings that discrimination set and other more general performance-set factors such as warm-up and learning-to-learn are significant sources of proaction in the present experimental design (Marshall, 1968).

Secondly, Group RCL should have performed less well than its appropriate ICL control if the learning of common labels to relevant stimuli effectively increases their similarity through verbally mediated equivalence. However, the performance of Group RCL was superior to that of ICL on all transfer trials. Superficially, these results are inconsistent with those of Katz (1963) and del Castillo \& Ellis (1968) who reported significant proactive interference and no significant proaction, respectively, when their RCL Ss were compared with $O$ controls on similar transfer tasks. However, the apparent inconsistency between studies is as likely to lie in the differing consequences of $\mathrm{O}$ and $\mathrm{I}$ control treatments in the $\mathrm{AB}-\mathrm{AC}$ paradigm as in the variable effects of $C L$ training with stimuli of different types, responses of different associative values, Ss of different ages, etc. In future predifferentiation studies using associative transfer tasks, the comparison of the consequences $I$ and $O$ conditions should quickly clarify their differential significance. The importance of the observed difference between the performances of RCL and ICL groups in the present study is that proactive facilitation has been observed following a procedure previously assumed to promote only interference.

Third, while Group RCL made fewer errors than Group ICL, its performance was nonetheless significantly inferior to that of Group RDL on seven of 20 transfer trials. Learning a distinctive label for each stimulus thus proved more facilitative than learning two labels to clusters of three stimuli. The order of performance of Groups RDL, RCL, and ICL may be satisfactorily explained by combining the hypothesized effects of verbal and receptor-orienting cues in the manner suggested by Cantor (1965), or by invoking the idea that differential cue selection is guided by differential associative and response learning demands (Johnson, 1963). While the present experimental design provides no basis for comparing the values of these approaches, the data do confirm Johnson's (1963) observation that the number of verbal responses is an important variable in determining the nature of proaction in the PD paradigm. Additionally, the data suggest that it is on early trials of the transfer task that the differential effects of $\mathrm{CL}$ and DL treatments will be found.
Fourth, fast and slow PD learners did not differ significantly in their transfer performance, although fast learners made slightly fewer errors than slow learners in all conditions. If associative interference is affecting the transfer performance of Group RCL at all, these data are puzzling in view of the evidence from studies reported by White (1965), that fast learners tend to make more frequent associative interference errors than do slow learners. In light of this inconsistency, a more rigorous analysis of the consequences of RCL training is called for. This need is emphasized by the possibility inherent in the hypothesis that the proprioceptive consequences of receptor-orienting and verbal responses are both affecting the performance of RCL Ss. If this assumption is correct, RCL Ss are simultaneously reaping the benefits of acquired distinctiveness and suffering interference from acquired equivalence.

\section{REFERENCES}

CANTOR, J. Transfer of stimulus pretraining to paired-associates and discrimination learning tasks. In L. P. Lipsitt and C. C. Spiker (Eds.), Advances in child development and behavior. Vol. 2. New York: Academic Press, 1965. Pp. 19-58.

del CASTILlO, D. M., \& ELLIS, H. C. The role of response-produced cues in paired-associate transfer as a function of intralist stimulus similarity. Psychonomic Science, 1968, 10, 197-198.

DOLLARD, J., \& MILLER, N. E. Personality and psychotherapy. New York: McGraw-Hill, 1950 .

ELLIS, H. C., BESSEMER, D. W., DIVINE, J. V., \& TRAFTON, C. L. Recognition of random tactual shapes following predifferentiation training. Perceptual \& Motor Skills, 1962, 10, 99-102.

ELLIS, H. C., \& HOMAN, L. E. Implicit verbal responses and the transfer of stimulus predifferentiation. Journal of Experimental Psychology, $1968,76,486-489$.

ELLIS, H. C., \& MULLER, D. G. Transfer in perceptual learning following stimulus predifferentiation. Journal of Experimental Psychology, 1964, 68, 388-395.

JOHNSON, R. B. Recognition of nonsense shapes as a function of degree of congruence among components of pretraining task. Unpublished doctoral dissertation, University of Virginia, 1964.

KATZ, P. A. Effects of labels on children's perception and discrimination learning. Journal of Experimental Psychology, 1963, 66, 423-428.

MARSHALL, M. E. Some effects of amount of verbal pretraining and availability of verbal labels upon performance in a discriminative motor task. Perceptual \& Motor Skills, 1968, 26, 27-39.

NOBLE, C. E. Measurement of association value (a), rated association $\left(\mathrm{a}^{1}\right)$, and scaled meaningfulness $\left(\mathrm{m}^{1}\right)$ for the 2100 CVC combinations of the English alphabet. Psychological Reports, 1961, 8, 487-521.

ROBINSON, J. T. The effect of learning labels for stimuli on their later discrimination. Journal of Experimental Psychology, 1955, 49, 112-115.

SOMNAPAN, R. Development of sets of mutually equally discriminable random shapes. Journal of Experimental Psychology, 1968, 76, 297-302.

WHITE, S. H. Evidence for a hierarchical arrangement of learning processes. In L. P. Lipsitt and C. C. Spiker (Eds.), Advances in child development and behavior. Vol. 2. New York: Academic Press, 1965. Pp. 187-216.

\section{NOTE}

1. The author is indebted to Andrew Birkenmeyer for his invaluable assistance in obtaining and analyzing the data. 